\title{
Community Based Robot Design for Classrooms with Mixed Visual Abilities Children
}

\author{
Isabel Neto \\ INESC-ID, Instituto Superior Técnico, \\ Universidade de Lisboa \\ Lisbon, Portugal \\ isabel.neto@tecnico.ulisboa.pt
}

\author{
Hugo Nicolau \\ ITI/LARSyS, Instituto Superior \\ Técnico, Universidade de Lisboa \\ Lisbon, Portugal \\ hugo.nicolau@tecnico.ulisboa.pt
}

\author{
Ana Paiva \\ INESC-ID, Instituto Superior Técnico, \\ Universidade de Lisboa \\ Radcliffe Institute for Advanced \\ Study, Harvard University \\ ana.paiva@inesc-id.pt
}

\begin{abstract}
Visually impaired children (VI) face challenges in collaborative learning in classrooms. Robots have the potential to support inclusive classroom experiences by leveraging their physicality, bespoke social behaviors, sensors, and multimodal feedback. However, the design of social robots for mixed-visual abilities classrooms remains mostly unexplored. This paper presents a four-month-long community-based design process where we engaged with a school community. We provide insights into the barriers experienced by children and how social robots can address them. We also report on a participatory design activity with mixed-visual abilities children, highlighting the expected roles, attitudes, and physical characteristics of robots. Findings contextualize social robots within inclusive classroom settings as a holistic solution that can interact anywhere when needed and suggest a broader view of inclusion beyond disability. These include children's personality traits, technology access, and mastery of school subjects. We finish by providing reflections on the community-based design process.
\end{abstract}

\section{CCS CONCEPTS}

- Human-centered computing $\rightarrow$ Accessibility; Participatory design; - Social and professional topics $\rightarrow$ People with disabilities.

\section{KEYWORDS}

inclusion, social robots, visual impairment, participatory design

\section{ACM Reference Format:}

Isabel Neto, Hugo Nicolau, and Ana Paiva. 2021. Community Based Robot Design for Classrooms with Mixed Visual Abilities Children. In $\mathrm{CHI}$ Conference on Human Factors in Computing Systems (CHI '21), May 813, 2021, Yokohama, Japan. ACM, New York, NY, USA, 12 pages. https: //doi.org/10.1145/3411764.3445135

\section{INTRODUCTION}

Blind and visually impaired (VI) children are increasingly educated in mainstream rather than special schools [24, 27, 48, 51].

Permission to make digital or hard copies of all or part of this work for personal or classroom use is granted without fee provided that copies are not made or distributed for profit or commercial advantage and that copies bear this notice and the full citation on the first page. Copyrights for components of this work owned by others than ACM must be honored. Abstracting with credit is permitted. To copy otherwise, or republish, to post on servers or to redistribute to lists, requires prior specific permission and/or a fee. Request permissions from permissions@acm.org.

CHI '21, May 8-13, 2021, Yokohama, Japan

(C) 2021 Association for Computing Machinery.

ACM ISBN 978-1-4503-8096-6/21/05 . .\$15.00

https://doi.org/10.1145/3411764.3445135

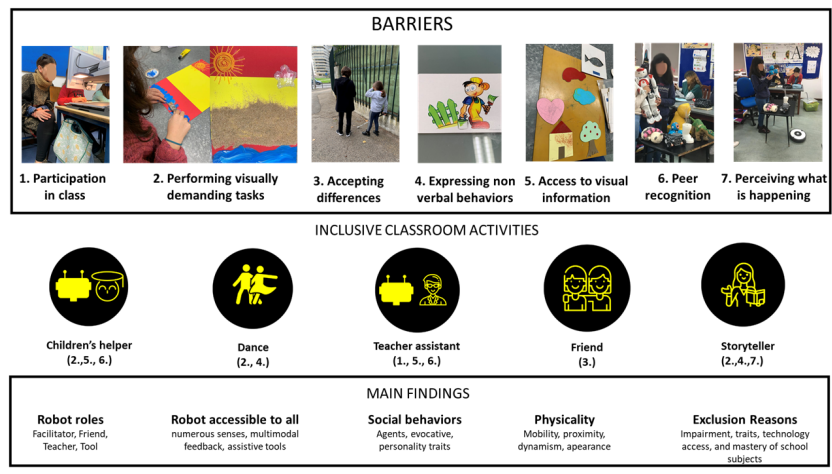

Figure 1: Robot design for Children in Mixed Visual Abilities Classrooms (Barriers, Classrooms activities and Findings)

Instead of viewing visual impairment as an issue to be fixed, educational institutions are adopting the social model of disability by focusing on reducing or removing the barriers that these children face [51,54]. A variety of stakeholders, including educators, teaching assistants, parents, and technologists are exploring new ways of holistically support VI children in their school activities [11, 17, 21, 52, 56, 56, 62, 64].

Current accommodations in mainstream classrooms include, for example, having a dedicated teaching assistant who sits with VI children through all classes and supports their learning activities [51]. Classroom content is usually made accessible through Braille, tactile diagrams or by using assistive technologies such as screen readers or screen magnification software. However, existing practices and technologies tend to prioritize accessibility over inclusion; that is, they are designed to be used by children with visual impairments alone and not by their sighted peers, which can reduce opportunities for inclusive classroom experiences. Indeed, recent studies show that children with disabilities face issues related to classroom participation, lack of collaborative learning, reduced social engagement, and potential for isolation [49, 51, 75].

Social robots could reduce barriers to inclusion. Robots are endowed with physical presence and possess social qualities that enable them to communicate with humans by following tailored social behaviors and rules. They are physically embodied and can combine several sensors as well as multimodal feedback capabilities, opening novel opportunities to enhance inclusive education where children with and without visual impairments can share technology $[49,58,75]$. However, this potential remains largely 
untapped. Although robots can be engaging to children [1], prior research has only started to explore robots as accessibility tools $[49,58,75]$ with very little work done on the co-design of social robots, particularly with mixed visual abilities children. This raises the need to explore the design of robotic devices that support interaction between disabled and non-disabled users in general, and poses a challenge for the design of inclusive experiences for mixed visual abilities children in mainstream schools.

In this paper, we explore the extent to which social robots could support inclusive classroom experiences, particularly by identifying what activities are most relevant, and what forms such support might take. We conducted a four-month long community-based design process through a multiple methods approach including ethnographic observations, contextual inquiry, group and individual interviews, and participatory design activities. We primarily engaged mixed visual abilities children as well as other stakeholders within the school community (primary and secondary education), namely educators, teaching assistants, special needs professionals, and parents. We thus aim to extend current work in accessibility and inclusive technologies by focusing on co-designing social robots in mixed visual ability settings and with a mix of stakeholders. We aim to answer three main research questions: (1) what barriers to inclusive classrooms can social robots address? (2) what characteristics do children with mixed visual abilities expect from social robots? (3) how can we engage children with and without visual impairments in participatory design activities of novel robotic devices?

To answer these questions, we conducted a community-based design process, starting with community engagement efforts to build connections with multiple stakeholders within a local mainstream school. These connections supported trust and relationship building, particularly with children and educators, creating a safe environment to exchange ideas before advancing into formal design activities. We were also able to better understand the dynamics and challenges of mixed visual ability classrooms and collaborate with educators to embed design activities within the school curriculum. We then engaged children in a four-week long participatory design process that included exploration, critique, ideation, making, and enacting activities. From this design process, we derived a set of recommendations for the design of social robots that support inclusive classroom activities.

The key contributions of this paper are: (1) identification of barriers experienced by VI children in mainstream schools and how these relate to social robots through novel interaction scenarios; (2) new design guidelines that contextualize social robots within inclusive classroom activities; (3) reflection on the challenges and benefits of engaging in a community-based design process with mixed visual ability children. These contributions are relevant to accessibility researchers and designers of technologies for groups with mixed visual abilities, particularly in the field of inclusive education. They provide a basis for designing support for inclusive classroom activities through social robots.

\section{RELATED WORK}

We discuss the related work in four fields of research: inclusive education technologies, robot technologies for visual impairment, robot technologies in schools, and inclusive, participatory design.

\subsection{Inclusive Education Technologies}

Studies showed that children with special educational needs, namely visual impairment, increased in mainstream schools [24], demanding new types of support, learning experiences, and social dynamics [14, 48, 54]. New assisted educational technologies are rising to meet this trend. Current assisted education technologies [6], such as amplifiers and braille writing machines, mainly focus on individual needs, frequently are complex and need additional support form a sighted person, and are often cost-prohibitive devices. Due to that, VI children are often excluded from classroom dynamics, learning in their pace [51].

Previous research has explored how novel interactive modalities, such as sound, haptic and tangible information, influence visually impaired children in the way they perceive visual information, geography, and diagrams [10, 16, 17, 26, 47]. However, schools are demanding new inclusive technologies and teaching approaches to allow full participation, regardless of children' needs [51]. Simultaneously, technology has become more accessible to the entire class, with digital whiteboards, mobile computers, and phones. Those technology-enabled learning tools could leverage to meet all learners' needs as new inclusive, affordable, and straightforward solutions. Some inclusive educational technologies are already rising for knowledge sharing [52, 58], and leisure [49]. Our work aims to enrich inclusive education techniques by using affordable and simple robots to foster all learners' participation in mixed visual abilities classrooms.

\subsection{Robot Technologies for Visual Impairment}

Physical embodiment, mobility, speech, and vision are capabilities that the robots have that fostered their use as assistive technologies. Previous research used stationary and mobile robots to help visually impaired people for everyday tasks, as an audio-vision translator for navigation [2, 40, 59, 74] or for color-mapping [63], or for manual activities, such as in Bonani et al. [13] work, where a social robot engaged and supported visually impaired adults through a collaborative activity associated with a physical assembling task.

More recent research started to use robots as social enablers, leveraging on their capacity to express and perceive emotions while sustaining social relationships [30]. In [8] a set of specifications were proposed on how to create a service robot that can interact and guide a blind person through a building in an effective and socially acceptable way.

However, to have broader use, some challenges need to be surpassed. Most social robots are costly, designed for adults, and to be used mainly in indoor environments. In order to have the required capabilities they are often endowed with perception capabilities and overloaded with multi-sensory information [22], making them complex to use. Furthermore, they are often designed for general application settings, and thus not adapted to the requirements and setting designed for VI children. To tackle those challenges, we aim to explore affordable robots (using off-the-shelf robots or DIY prototypes) adapted to each child's ability.

\subsection{Robot Technologies in Schools}

Robots are starting to be used in schools especially when there is a need for physical interaction [12,61], like exploring navigation 
skills, tutoring physical exercise [44], learning handwriting [7, 34] or geometry [58]. They can also explore physical embodiment and visual-spatial working memory with children with visual or hearing impairments [11, 25, 39, 49, 58, 75]. Educational robots can play different roles in schools, such as tools, teachers, or peers, having shown their ability to support learning activities, help assisted therapy, or improve social interactions. [12, 38, 70]. They can focus on the learning of a particular subject, and foster engagement [1] in that process. They are ideal for supporting new educational methods, like exploring learning by teaching [37] or applying artificial intelligence techniques to personalise and adapt to the students' needs. However, the potential for addressing inclusion by using social robots in mixed visual abilities classrooms deployments remain, so far, fairly unexplored.

\subsection{Inclusive Participatory Design}

Assisted technology tools, namely for visual impairment, are often designed by sighted adults, that develop products and services to overcome the accessibility gap, without counting on the age of the user, interest, and brain ability to perceive that information [22, 78]. As a result, there is a lack of use of these tools by children.

Previous design sessions already included visually impaired individuals $[8,50,69]$. Moreover, children were also involved in designing robots, $[3,60]$. In recent research, mixed-visual ability children played as creative agents in designing technologies for inclusion in schools [21, 55].

Design methods often depend on visual information, memos, drawings, and role-plays, which can be a barrier for visually impaired and young children. In an inclusive, participatory design, cross-modal interactions, to allow synchronous information sharing in multi-sensory ways, are essential to retrieve and share information between young participants [50,55]. For instance, in Metatla and Cullen's work, [21, 49], they explored tangible approaches using robots, Metatla $[50,52]$ also used audio-tactile physical mock-ups designs, and Ibrahim et al. [35] used documentaries in the design process of technologies for disability. Children widely use role-play and body storming techniques in design [3, 18, 55, 71], allowing them to act and behave according to a specific context. Furthermore, the expanded proxies technique helped children empathize with each other differences in one design process to tackle inclusion [53].

Similar to Metatla et al., we are working with mixed-visual ability children, acting as creative agents in co-design activities to the design of technologies to foster inclusion in schools.

This paper extends the prior work in inclusive design by including the design activity in a mainstream school curricula in three classrooms with mixed-visual ability children. In that way, different school levels (primary and lower secondary education) worked with us, allowing us to explore the impact of age in design activities and children's perception of inclusion.

\section{COMMUNITY ENGAGEMENT}

All the children and school staff were selected from a local inclusive school and accepted to participate in the study. Parents and school staff gave their informed consent for all the sessions and the use for academic purposes. The local university ethical committee approved the study and the methodology.

The first stage of our research was centered around building relationships with stakeholders of a local school community, including educators, children, teaching assistants, special needs professionals, and parents. The aim was to build meaningful connections, mutual understanding, and trust to create a safe environment to share ideas and concerns as we progressed in our research. Thus, we engaged in a four-month-long community-based design process. The school is a privately funded institution for children aged 0 to 15 years old, which includes nursery (0-2 years old), kindergarten (3-5 years old), primary education (6-10 years old), and lower secondary education (11-15 years old). We focused our research efforts in primary and lower secondary children by immersing ourselves in multiple school activities to learn about inclusive mainstream schools in general while engaging with all stakeholders. This research stage also allowed us to address the first research question: what barriers to inclusive classrooms can social robots address? The goal was to understand mainstream schools and the challenges faced by VI children and educators in these settings.

\subsection{Methodology}

We combined multiple research methods to address the research question. We engaged in classroom observations in four different classes with mixed visual abilities. Each classroom had one child with visual impairment (one blind, three low vision) and sixteen sighted peers. In total, we observed 11 lectures of different subjects, which corresponds to 14 hours of observational data. During such observations, we often coordinated with educators to participate in classroom activities such as group readings and games. This allowed us to begin building rapport and fostering relationships with children, educators, and teaching assistants. We observed classrooms with and without teaching assistants to learn about VI children's experiences when specialized one-to-one teaching is not available in the classroom. We also interviewed four educators and one teaching assistant to understand better the challenges and educational strategies currently used in mixed visual ability classrooms.These interviews lasted between 15 to 30 minutes and explored four main questions: What are the educators' main challenges in a mixedvisual ability school? What are the children's challenges? What are the critical barriers of children in group activities? Moreover, how can robots help overcome these challenges?

For expert opinions on the challenges of special needs education, we conducted 19 contextual inquiry sessions with four professionals: braille teacher (four sessions, two children, one blind and one low vision, total of two hours), navigation and mobility instructor (five sessions, four children, one blind and three low vision, total of four hours), speech therapist (nine sessions, six children, two low vision, total of 4.5 hours), and psychologist (one session, one hour). These professionals are part of the school community and provide support and complementary education to children with special needs, namely VI children. The goal of these sessions was to experience its dynamics and challenges as well as to understand what tools were used.

We also interviewed six parents to learn about their lived experiences raising and educating a child with visual impairment. 
Each interview took, on average, 75 minutes. We explored four questions: What are your main concerns as a parent? What are the main challenges perceived by your child? What are the main challenges of your child in group activities in classrooms? and How can robots help overcome those challenges? They also served as proxies to uncover negative and positive experiences of VI children in (and outside) mainstream schools. Simultaneously, we could leverage the connections with parents to engage with two different local associations that support VI children and their families. We interviewed six people who acted as directors or coordinators of these institutions and promoted multiple social inclusion projects for children with mixed visual abilities.

After four months of engaging with stakeholders from a local mainstream school, we understood the community as a whole better and identified existing barriers to inclusive education. Overall, we were able to engage with more than 90 children (seven VI), aged between 6 and 15 years old, and collected more than 40 hours of interviews with educators, teaching assistants, special needs professionals, and parents. We used grounded theory [19] to identify the emerging themes showing the barriers to inclusive education in mixed visual abilities classrooms. One researcher created the initial codes and an affinity diagram of the main findings. We conducted peer validation throughout the coding process [15], where three researchers met regularly to review and clarify coding and grouping decisions. We determined general themes through iterative discussions.

\subsection{Barriers to Inclusive Classrooms}

Stakeholders provided multiple perspectives about the experiences of children with visual impairments, depending on their community roles. Discussion were geared towards children's activities and technology use in schools. Here, we focus on the most relevant barriers as they relate to the capabilities of social robots.

Children participation in class. In classrooms, educators focused on classroom dynamics, leading group interactions, while reaching individuals from time to time to teach or ask something. One challenge for an inclusive classroom was to balance children participation and engagement, creating a secure environment for all children and encourage them to self-express. When the teacher assistant (TA) was in the class, this balance was harder to reach, the principal teacher focused on the rest of the class, and TA frequently focused on his own VI child. While addressing VI child needs, to keep the learning pace of the class, TA sometimes ignored the class dynamic, leading to a separate way of learning and the VI child lack of participation on the group activities. Fig. 1, shows a VI child focused on an amplifier, ignoring the rest of the class, with a TA on her side. One teacher suggested using a robot as an engagement controller. These robots could help the teacher balance children's participation by perceiving and measuring all children's engagement (by counting children's interactions) and identifying the next speaker.

Performing visually demanding tasks. One barrier observed for inclusion in mixed visual abilities groups is that people tend to avoid asking VI children to do visually demanding tasks, without exploring any other way to overcome their difficulties. Although this is understandable while mitigating the risk and minimizing frustration. It sometimes leaves unexplored paths for innovation, reducing future expectations. Some parents, educators, and even classmates stated that sometimes they constrain VI children's jobs to mitigate their frustration of not doing a specific task. For example, they avoid asking a VI child to do a visual task, such as reading a book, doing a puzzle, getting a rubber, finding an answer in a text, or drawing a picture (fig. 1 shows a blind child painting process). Without facing these challenges, VI children often do not explore new ideas and solutions, impacting inclusion in classroom tasks. Innovation in visual recognition shows a considerable potential to use technology, and social robots, to be people's eyes $[2,13]$. In reading and exploring text activities. Robots could take a photo of the text, convert it to non-visual interfaces, such as sound, braille, or digital information and allow VI children to access the text and explore it from anywhere. Educational teacher and the braille teachers also suggested that robots could help visually impaired children dance and paint by helping them perceive the space, movement, and the drawing result.

Accepting differences. Parents, educators, and psychologists referred to as common challenges children's acceptance of their impairment. All VI children involved in this research found it difficult to accept their differences (in fig.1 a VI girl was walking in the street, and was trying to hide her walking stick), deal with the frustration, and have or had therapy to overcome that feeling. Two of them also practice yoga or judo to help manage stress and improve their body space awareness. One mother referred that outside the school, there is a clear sense of exclusion from sighted children (and even adults). People in general do not know how to talk with her blind child, avoiding any contact, which leads to a powerful feeling of exclusion from her daughter and herself. This discomfort is well known in literature [68] was also mentioned by one of the Braille teachers.

One future use of social robots could be to help children overcome their frustration by playing the role of a personal confidant. Therapists and the psychologist involved in this community study, suggested a potential use for robots in their sessions could be impersonating a "blind" robot that needs to overcome some difficulty, and thus helping by seeing it through a third person perspective. However, the psychologist involved in this study also raised some concerns on the impact of involving a robot in the therapy, particularly in using robots as friends' substitutes, which could have a negative effect by reducing the need for peer interactions.

Expressing non-verbal behavior. In our research, we interviewed two braille teachers. They were both visually impaired: one was blind and the other with a profound low vision. These sessions were very enlightening, as both of them had been VI children, they shared with us their feelings and frustrations as they grew up. They detailed several challenges for VI children. They pointed out that one of the major challenges for inclusion where robots could help lies in the difficulty that blind children have to learn facial expressions and social gestures, like looking surprised (open their eyes), saying ok, or thumbs down. In general educators avoid using visual cues, limiting the communication and learning of those cues. Due to that, blind children regularly do not use gestures (facial or body movements) to express themselves [31, 46] affecting 
the interaction since they usually remain in the same pose and expression. In a mixed-visual ability group conversation [68], it is sometimes tricky for sighted and visually impaired children to perceive each other emotions. Following the braille teacher suggestion, social robots could help address this challenge by recognizing their peers' non-verbal behavior and imitating (physically) or verbalizing it, thus helping a VI child express and change their non-verbal behaviour accordingly.

Access to visual information. Classrooms are full of visual information. educators referred that they try to translate all visual information there present and share it with their pupils. However, there is always some loss of information and a delay in sending and receiving that information. One example given occurred in a math class, where the teacher was explaining some geometry concepts and used her body movements to illustrate the difference between parallel and perpendicular lines. The VI child in the classroom was obviously not able to perceive the gestures. She suggested that a robot could help by mimicking her movement to help children would physically, through touch, follow the robot's gestures, and thus learn spatial concepts and geometry in the future.

Peer recognition. Blind and low vision children need to continuously identify their peers and educators based on sound and other clues, which can sometimes be impossible due to overlaps in sound or distractions. It is a considerable barrier to inclusion, as the VI child sometimes does not know with whom he or she is interacting with. Educators referred that robots could support VI children during classes to help them by moving in the direction of the speaker, or naming her/him, helping to identify who the child is interacting with.

Perceiving what is happening. A mother referred that VI children often ask some sighted peers to describe the environment. Although many children help, this often becomes hard to sustain. She also gave how this affects the inclusion in family activities. One example shared was related to a family outing to see a ballet show. Her VI child was repeatedly asking her mother to describe what was happening. After a while, both were bored and exhausted, and stopped sharing what was happening; they never went to see a ballet show again as a family. She said that it could have helped blind children perceive the performance autonomously and return to ballet again if they had a robot that could also help by describing what was happening. Figure 1 shows examples of classroom activities associated with this barrier.

The stakeholders raise novel uses of social robots to overcome physical impairment challenges at school and society. They can play a role in enabling VI children to be more involved in classroom activities, to know who is participating and perceive their behaviors, to perceive spatial concept, convert a text to non-visual information on the fly (without the need of waiting for a braille version or larger font). Robots can also be used in therapy to proxy a situation or describe a family event or a classroom activity. However, educators also fear the impact of robot dependency for a visually impaired child's future relations with other children.

\section{INCLUSIVE PARTICIPATORY DESIGN PROCESS}

Having gained an understanding of the barriers in mainstream classrooms and identified potential activities for social robots to promote inclusive education, we aim to address the second and third research question: what characteristics do children with mixed visual abilities expect from social robots? Moreover, how can we engage children with and without visual impairments in participatory design activities of novel robotic devices?

We conducted a four-week long participatory design process at the same mainstream school, where we engaged with three groups of mixed ability children once a week. The groups were selected based on one VI child's presence in their class, so all the participants were used to work in mixed-visual abilities groups. Based on the feedback from educators and school coordinators, we felt necessary to engage with the whole class to guarantee that all children would be included in the activity rather than just a subgroup selected based on physical abilities or other factors. The inclusive, participatory design process was embedded in the school curricula, particularly in the technology education classes and citizenship classes. Together with three educators, one per each school level, and a teaching assistant, we created a series of workshops and activities to design robotic devices for inclusive education. We conducted these activities with 54 children ( 5 visually impaired) from primary and lower secondary education.

\subsection{Classrooms activities for Social Robots}

For each of the barriers we explore different activities with robots and five come up: (1) Child helper for supporting VI children to perform visually demanding tasks, and access educational information in an autonomous way; (2) Dance, to foster the exploration of non verbal behaviors, and space concepts in a visually demanding task; (3) Teacher assistant, to help children access to information and participation in classroom activities, (4) Friend, to promote relations that accept, value and adapt to each other differences and behaviors; and (5) Storyteller, to explore acting and non verbal behaviors. In fig 1, show the relation between emerged barriers and activities for the participatory design. Three principal educators reviewed the activities proposed to ensure that those were inclusive and relevant in the school context.

\subsection{Inclusive Prototyping Toolkit}

One of the objectives of the workshops was for mixed-visual ability groups to build physical prototypes. We paid special attention to the accessibility of materials for the design workshop, including using large fonts, Braille, color-coding [57], and textures (Figure 2).

To help the ideation processes, we printed cards with features of social robots as well as blank cards that children could use to propose novel features. Each card included an image, printed labels, Braille labels, and texture information. Cards were also color coded by features: communication, emotion, personality, mobility, material, senses, functions, and shape. We identified several options for each of the robot's features, as illustrated in Figure 2. Blank cards for mobility, shape and function, for them to define. And We created five predefined features values inspired by our community engagement process. Communication cards: (1) Writes, (2) 


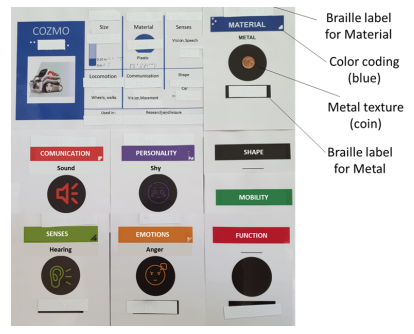

Figure 2: PD toolkit - example a file for cozmo robot and feature cards

Body Movement, (3) Speaks, (4) Sound, and (5) Facial Expressions. Emotions printed cards were seven [28, 29, 32, 33] : (1) Happiness, (2) Sadness, (3) Calm, (4) Nervous, (5) Anger, (6) Excitement, and (7) Furious. Personality cards were inspired by the Big 5 classification model [20], resulting in eleven cards: $(1,2)$ Curious or Disengaged (openness to experiences); $(3,4)$ Nervous or Calm (neuroticism); $(5,6)$ Outgoing or Shy (extraversion); $(7,8)$ Organized or Messy (conscientiousness); $(9,10)$ Nice or Bad temper (agreeableness) and (11) No personality. And material cards were: (1) Fur, (2) Plastic, (3) Metal, and (4) Tissue.

\subsection{Participatory Design Process}

We ran a four-week long design process where we held a series of design workshops to generate insights designing social robots for inclusive education. We worked with 54 children (5 visually impaired) divided into three groups, which corresponded to their school year: Primary school $(\mathrm{N}=18,3$ children with low vision, Age $=8.55, \mathrm{SD}=1.29)$, fifth grade $(\mathrm{N}=19,1$ child with low vision, Mean Age $=10.78, \mathrm{SD}=0.41)$, eigth grade $(\mathrm{N}=17,1$ blind child, Mean Age $=13.23, \mathrm{SD}=0.43)$. We split the groups into smaller teams of 3-4 children. These teams remained the same throughout the design process. The workshops took place during a normal school day and were part of the class curriculum.

The design process followed five phases: (1) exploration and familiarization, (2) activities and ideation, (3) presentation and critique, (4) making robotic devices, and (5) enacting interaction.

In the exploration and familiarization phase, we gave an informal oral presentation about robots and show a set of robotic devices with multiple characteristics and designed for different purposes. The goal was to familiarize children with robotic technologies and demonstrate their physical capabilities. We showed 11 robots alongside a printed description card that included information about each robot's size, shape, material, sensors, movement capabilities, and communication capabilities. Cards were Braille labeled and included a sample of the robot's material (e.g., metal plastic, fur) (Figure 2). The cards were used in the following design phases and worked as inspirational materials for children's designs. The 11 robots were: Cozmo, Roomba, Keylo, Lego Mindstorms, Yolo, Dash, Pleo, Sphero, iCat, Nao and a soft toy that simulated being a robot. $[4,5,36,41,43,65,66,72,73,76]$. These robots were not used in the following sessions.

In the activities and ideation phase, we randomly assigned an activity to each team and encouraged the children to discuss and detail a activity for each activity. We used five activities: helping children, dancing, helping educators, being a friend, and storytelling. We then asked children to begin discussing how a robot could be used in such a context. The facilitator guided children through a series of questions such as What would be robot's role? What would it do? How would the robot do it? What characteristics shout it have? We used the inclusive prototyping toolkit described in the next section to engage children in idealizing a robotic device. In addition to the available materials, children could create new features, functionalities, or characteristics they envisioned the robot performing. Participants were strongly encouraged to work as a team by proposing, sharing, and building on each other's ideas. The educators had an important role in helping the research team in moderating and guiding discussions. Researchers made sure children with visual impairments understood the inclusive prototyping toolkit and all the available materials. During group work, we made sure all children had an active voice in the activity. Educators had the vital role of directing questions to the least participative children and supporting them in expressing those ideas (e.g., by writing or reading post-its on their behalf). Researchers also had an active role by prompting children to build on each other's ideas or think about alternatives. At the end of each session, all children, including VI children, presented their ideas to the class.

The presentation and critique phase took place once children finished idealizing their robots. Teams were asked to share their ideas and design decisions with the class. Other children were encouraged to comment, ask questions, or provide new ideas to their classmates. The facilitator also provided several reflective questions to understand why children chose, for instance, a given morphology or communication modality.

Next, we conducted the making robotic devices phase. Unlike the other phases, which took place in a single design session, over 3 weeks. Teams were asked to build the robots. They idealized robots using recycled materials. The goal of this activity was to encourage children to materialize their ideas and reflect upon it. Additionally, they could use the physical prototype to illustrate behaviors and bodystorming $[18,53,71]$.

The final phase of the design process was enacting. Children were asked to create a script that demonstrated how their creation would behave in the proposed activity. Children were encouraged to enact a small play to illustrate the capabilities of the robot in group interaction. There was a strong emphasis on asking children to simulate what the robot would do and how it would do it, particularly how it would interact with others.

During four weeks, we organized weekly workshops, of two hours, for each of the groups, organized as follow : Workshop 1 In the first week, children did the activities and ideation and presentation and critique phases. Workshop2 In the second week, we started the making robotic devices phase. children focused on detailing their group ideas, selecting the final features, materials, and drawings. They shared and presented a detailed design in an online class. Workshop3 In the third week, children continued the making robotic devices phase. They made their robotic devices individually, and showed their prototypes in an online class. And Workshop4 In the fourth week, the enacting phase took place. A role-play with the robot prototype, was recorded in video and presented to the class. 


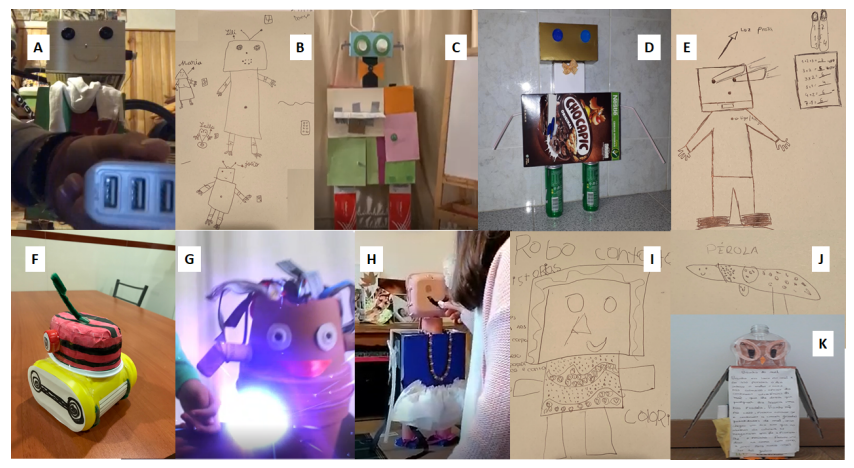

Figure 3: Robots prototypes co-design with mixed-visual ability children

\subsection{Findings}

This section presents the analysis and results of the fifty-four drawings and class presentations, twenty-four physical prototypes, and fifteen role-plays. All classes involved had one VI child, and all participants were familiar with interacting in mixed-visual ability groups. Figure 3 illustrates examples of robots drawing sketches, prototypes, and role-plays. Robots C, D, E, F, G, I, J were designed by mixed-visual ability team members, robots $D, J$, G were built by VI children, and sighted children built the remaining robots. All teams shared their ideas in the class, and received feedback from their colleagues. We had three teams for each activity child helper $(\mathrm{N}=9$, one visually impaired); Dancing $(\mathrm{N}=10$, all sighted); Teacher assistant $(\mathrm{N}=12$, one blind, one low vision); Friend $(\mathrm{N}=12$, one low vision); Storyteller ( $\mathrm{N}=11$, one low vision). In this section we detailed the main findings that emerged from the data.

Educational robot's roles to foster inclusion. Overall, the diversity of inclusive experiences in schools prompted participants to produce robot designs with a range of roles, commonly used in education [12, 38, 45], such as facilitator, friend, teacher and, tools. However, they were distinct, from other educational tools, in three ways: They were designed to circulate, had several assistive tools, and focused on children' engagement $(\mathrm{N}=30, \mathrm{SD}=0.49)$ rather than in one to one interactions $(\mathrm{N}=14, \mathrm{SD}=0.44)$.

Facilitators robots were agents supporting the activity $(\mathrm{N}=30$, $\mathrm{SD}=0.50)$.The "CACf robot $(C, D)$ ", were two teacher assistants, made by one blind and one sighted girl from the same team. They were able to do repetitive tasks, such as repeating the previous teacher information, when children were inattentive or keep silent in the class, and had a braille machine and an amplifier to extend VI children capacity. "Joca robot $(F)$ ", from a sighed team, is a children helper robot. Provided alerts, to keep children aware of time, and had also mobility enhanced ability's to climb the stairs to move around to collect materials or share messages between classrooms. On the other hand, "Mr. Owl robot (L)", a storyteller robot, was made by a sighted team. It focused on accessibility in performative experiences in reading and performing stories to children. The owl robot understood lips movement, and sign language wrote the story in the belly and moved wings to show the story pace.
Friend robots were designed for social engagement and companionship $(\mathrm{N}=21, \mathrm{SD}=0.48)$. The "Jaquim robot $(A)$ " was made by a sighted team, acted as a friend in the play-yard. He took photos and selfies with children and offers them cookies to raise new friends. It also had facial recognition capability and long arms to hold lonely children, and guide them through the play-yard to find their friends. The "Roxy robot (I)", was made by a mixed-ability team an acted as a companion. It fostered each child well being, and cheer-them up by eating child's bad feelings. It was also enriched by voice recognition to identify each child and adapt behavior accordingly to their emotions and traits. Two girls from the primary school level were in the initial reading phase; one of them had visual impairment. The "Tale teller $(\mathcal{F})$ " and "Pearl $(K)$ " robots were actors performing stories, they projected stories through their eyes and use color lighting to classify the story complexity.

Teacher robots had agency in the learning process, playing as educators or tutors in classrooms $(\mathrm{N}=18, \mathrm{SD}=0.47)$. "the fo, Lili, Mary, Laila and John (B)", performed by a sighted team, as a dance group, taught dance space, expressions through body movements and rhythm. They played as principal teacher, whenever "human teacher" was absent, or as dance partners. Children could interact with them by pressing a button in the belly to play music and select the dance to perform. "Teacher Anna robot" (E), was made by a mixed-visual ability team, she was just like her teacher "Anna," that moved around the class, reinforcing principal teacher information to the all-class or to small groups. By zooming and explaining doubts in a mobile tactile board in any place of the room. During the role-play phase, robots were also used at home when children were in remote education mode. They played as tutors and helped children do their home works or studying, and at teacher's home, they were used to validate children' work.

Tool robots, participants designed robots as tools $(\mathrm{N}=10$, $\mathrm{SD}=0.38$ ). They use robots as tools providers, such as translators, cleaners, or logistics machines. They differ from facilitator robots, as they were executors that reacted to children's orders; they did not act in any proactive behavior. "RAVI robot $(G, H)$ ", made by a mixedability team that loved music and dogs; RAVI robot interacted by speech and sound and had the ACDC main singer's voice. It also understood and spoke in animal language, so it played as a translator machine, allowing the group to talk and interact with dogs. RAVI was also a logistic machine used around the school, exploring robots' physical capacity to move, hold and manage proximity to preserve children and teacher personal spaces. It had a Caterpillar and hooks to climb and collect an object from outreached places. Although it had a personalized voice to interact and the capacity to talk with dogs, it did not trigger any action by itself; it only reacted to requests. At home in role-plays, those tool robots also performed as bedroom cleaners (tidying up toys) and sanitization machines, due to COVID pandemic impact.

Robot accessible to all. Children designed their robots to be accessible to all, with numerous senses to adapt to children' needs, multimodal feedback, enriched by several assistive tools.

Children selected vision as the most used robot sense $(\mathrm{N}=39$, $\mathrm{SD}=0.45)$, followed by hearing $(\mathrm{N}=38, \mathrm{SD}=0.46)$, and touch $(\mathrm{N}=15$, $\mathrm{SD}=0,45)$. No one enables their robot with taste or smell capabilities. Vision and hearing coupled together and used simultaneously to 
enrich information, for example in "Jaquim robot (A)"'s roleplay, children asked "Faquim" to "find my friend," and the robot used vision to look for him in the play-yard. Touch was used to perceive child emotions in hugging, or to receive orders with buttons. Only one robot did not combine multiple senses and used a keyboard to input information.

All robots had more than one output channel. They vary between the human-like channels $(\mathrm{N}=34, \mathrm{SD}=0,49)$, such as sound, speech, body movements, facial expressions, haptic, and tactile; and extra-human channels $(\mathrm{N}=21, \mathrm{SD}=0,49)$ such as lighting, projection, inter-robot communication, animals language, power line or out of human-hearing frequencies. In the performative activities, dance and storyteller, the most used output channel was the visual $(\mathrm{N}=8, \mathrm{SD}=0.63)$, due to body movement and facial expressions. All other activities, the sound was the primary communication channel $(\mathrm{N}=43, \mathrm{SD}=0.90)$, due to its ease of use and its potential for engaging all children of this group. For robot visual feedback, coloring and lights were the most used ones $(\mathrm{N}=21, \mathrm{SD}=0.49)$ to focus attention and awareness on information shared, exploring robot physical dynamics to perceive space, position, and movements. All VI children involved in the study could perceive lights (and two were color blind), so the sighted and mixed-visual ability teams were familiar to use amplifiers, colors, textures, and lights to share information inside the classroom.

The children explored several different accessibility features and tools; 34 prototypes had at least one assistive tool $(\mathrm{N}=34$, $\mathrm{SD}=0.52$ ). Sound and verbal communication-based technology were the most commonly selected, in sync with preferred output communication channels $(\mathrm{N}=29, \mathrm{SD}=0.49)$. They were used to repeat teacher speech, or voice recognition. Children designed tactile based technology $(\mathrm{N}=5, \mathrm{SD}=0.29)$, such as braille printers, buttons, and tangible tablets. Furthermore, nine robots had visual-based technology $(\mathrm{N}=9, \mathrm{SD}=0.38)$, such as sign language, projectors, and face recognition. Furthermore, all of them had mobility.

Robots social, physical and intelligent behaviors to foster inclusive experiences. Children design robots as social agents $[9,23,30]$, to perceived and express emotions through natural clues. For example, "Roxy robot (I)", cheer-up children. Alternatively, as social evocative "Teacher Anna robot (E)" spoke softly, clearly, slowly, and to help children be focused and engaged in the classroom, allowing full participation of the all-class. They also performed distinct personalities. The most common traits were outgoing and happiness $(\mathrm{N}=32, \mathrm{SD}=0.50)$, but they also mimic or complement children' personalities and interests. "RAVI robots $(G, H)$ ", mimic team members' interests "our robot do not like football or do dangerous activities". Other robots complement team weakness "our robot will be organized, and cleans the room because we are messy".

Moreover, robot dynamics and physicality nurtured mixedvisual ability relations. Robots were using their mobility capacity to do actions by the children, allowing children to stay longer together, as in "RAVI robots $(G, H)$ " that collected missing school materials. They were also used, as in "Jaquim robot (A)", to create new children interactions, by leveraging robot proximity capacity, holding children's hand, and guide them around to approach new friends. Robot's appearance vary from objects, animals, humans, and fiction shapes [3,77]. Robots were all up to $1 \mathrm{~m}$ height, maybe to ease children interactions and possible support materials weight. In some cases, robots impersonate their friends or educators, "our robot is like our friend Sophie" or "our robot has our teacher's name, Anna, and size". Children also dressed robots up to express different personalities and explore different textures, using clothes, high heels shoes, and pirate hats.

Children designed intelligent robots for teaching roles, when robots helped children in their homework, or whenever they were proactive.

Inclusion through children eyes. When analyzing the data through children's eyes, we found out that the most common reason for exclusion was their state of mind (shyness, loneliness, or terrible temper) $(\mathrm{N}=17, \mathrm{SD}=0.64)$ and not impairment $(\mathrm{N}=11, \mathrm{SD}=0.36)$. In the third-place, they identify a lack of shared interests $(\mathrm{N}=9$, $\mathrm{SD}=0.6$ ). This finding suggests that children do not recognize impairment as the primary exclusion factor, even though teams frequently selected accessibility features for their robot. For primary school, child loneliness was the main exclusion factor; however, a girl, robot "Pearl (K)" fig. 3, added knowledge as a critical factor (she had difficulties in reading, and that made her feel uncomfortable and excluded). In the fifth grade class, the top exclusion reasons were different interests $(\mathrm{N}=9, \mathrm{SD}=0.59)$ and shyness $(\mathrm{N}=4, \mathrm{SD}=0.41)$. Only the oldest class mention impairment as the main factor for social disengagement, followed by shyness and loneliness $(\mathrm{N}=7$, $\mathrm{SD}=0.24)$. This finding suggests that age influences the perception of impairment as a factor for exclusion.

For children, the most relevant activity for inclusion was friend " all children need friends, to share their daily life, for support and companion, followed by teacher"Teachers need help when no one is paying attention", and children helper "child helper is useful when a child is ill, and a storyteller is relevant for promoting reading", the least relevant was dancing " all children know how to dance, we do not need a robot for that".

A child suggested at the end of the participatory design a genuinely inclusive activity, to be explored in future work. He suggested that "a robot could build a movie theater in anyplace in the school. A robot would project the film, mimic acting, describe the scene, and at the same time, convert the subtitles to native languages and sign languages. The class could select their favorite film and experience the movie together, allowing all children with and without impairments, to engaged in an extended reality experience".

\section{DISCUSSION}

This section discusses our research reflection, robot design recommendations, and limitations of our work, with an Inclusive school community. The barriers for inclusion in schools identified by the community and the analysis of the robot prototypes made by mixedvisual abilities teams can guide designers and researchers for future work with educational robots and design patterns for inclusion in human-robot interaction. Future inclusive, participatory design sessions can benefit from our embedded school curricula experience, dealing with different abilities, and reusing our inclusive toolkit for robot design. 


\subsection{Reflections on Community-based Design}

Findings from community engagement with educators and children through interviews and observations showed the richness and enlightenment of a user-centered approach to envision challenges for inclusion and how technology can tackle them.

Being present when working with a school community, is essential to foster trust and engagement with stakeholders. It allowed them to share fears, challenges, and experiences, during lessons, break time, or whenever they occur, without having a predefined slot for sharing all their thoughts in a one-time interview. Being in school allowed us to speak regularly with them. In some cases, they reflected on initial conversations and returned to us with new insights and thoughts, allowing us to have a broader view of inclusion challenges. We reached parents and learn about their family challenges daily. Talked with visually impaired adults, that shared with us their child experiences. Observed the classroom impact with and without special needs teacher presence. We spotted play-yard dynamics and discussed their fears and concerns with educators when dealing with mixed-visual ability children. Moreover, we perceived children's emotional needs by speaking to them and talking with parents, educators, therapists, and psychologists. Also, by observation of the school dynamics, researchers uncovered inclusion and exclusion behaviors. A child put his arms around a visually impaired peer to drive him around the class, or some derogatory comments on younger ages related to their colleagues' ability to read or draw. Researchers' role and ability to empathize with different stakeholders, adapting to children's dynamics, differences, and needs was also crucial for the engagement throughput [78] Moreover, It was of foremost importance to align the interviewees to the research's aim and show them novel technology to enlighten the interviewees to future robot uses.

\subsection{How to Engage in Inclusive Participatory Design}

Our design-based approach allowed us to discover novel ways to engage with mixed-visual abilities classes in an inclusive, participatory design while building social robots to tackle schools' inclusion. These sessions' findings are useful to others co-designing with visually impaired and sighted children, as they give insights into the challenges of an engaged inclusive, participatory design with children.

Embedding the participatory design in school curricula. We included our activity in the technology education discipline during lectures, in fig. 1 photos of two participatory design sessions. We enriched teacher educational material with our material. All materials were reviewed and aligned with educators, adapting our writing to children's ages, knowledge, and subjects. Researchers knew the participants and had previously been in those classes for over a month. This preparation phase allowed researchers to bond with the teacher, understand children's dynamics inside the classroom, and accessibility constraints. Moreover, at the start of participatory design activity, children had already engaged with researchers, accepting their authority, and co-leadership with their principal teacher. Educators and researchers were asked not to share their ideas, to mitigate the risk of restraining children's thoughts. One braille teacher printed all braille labels, and researchers and educators reviewed wording. The school community was tireless in supporting these activities, and their expertise, and insights were priceless.

Adapting materials and tasks to children's different needs without an individual approach. During the preparation phase, we created materials, built a PD toolkit, illustrated in fig. 2, and sessions logistics were adapted to classroom space. All materials were multi-sensory for VI children to perceive the information simultaneously with their peers and in most of the time in the same platform. During the participatory design sessions, researchers equally treated children. In some demanding visual tasks, such as exploring the robots, we asked visually impaired children to help and hold the robots while presenting them to the class, see photo in fig. 1. With this approach, visually impaired children could touch and calmly explore the robots before the others, without forcing an individual task. Whenever there was a specific need from a visually impaired child, not solved inside his team, researchers and educators addressed that as a group task.

From a community-based approach to a participatory design in the context. Our novel approach of a PD within a classroom context was possible due to the previous community engagement process. In the first stage, the stakeholders acted as informers [42], which allowed the gathering of the barriers to tackle and use in further participatory design activities. At the same time, it enabled researchers to build rapport with school stakeholders, namely educators and children. In the second stage, the participatory design process was co-designed with educators; that is, design research activities were embedded in a multi-learning experience [42], where educators shared pedagogical goals and researchers contributed with design methods to achieve those goals within a classroom environment. The participatory design process was structured around pedagogical goals that ranged from understanding and reflecting about challenges, working in groups to ideation, researching, prototyping, and communicating ideas. Moreover, these activities were created within the classroom context with situated challenges [67]. Children's living experiences in mix-visual ability schools and inclusive co-design activities, resulted in social robots that are inherently accessible or aim to tackle accessibility issues in this context.

\subsection{Design Recommendations for robots for inclusive classroom experiences}

Our analysis identified robot design features and children's perspectives for inclusive activities. In this section, we integrate these findings and discuss how children perceive the need for a robotic device for inclusion. Understanding these needs can guide robot designers when addressing robot for inclusive experiences.

Robot physicality over social and intelligent capabilities. We identify design recommendations for robots using their physicality, social capabilities, and intelligence. Physicality of the robot, is the primary feature to foster engagement in mixed-visual ability children, allowing children to have a holistic tool that moves in the school, and supports them in classroom activity, allowing 
them to interact with it wherever they were and whenever they want. Robots often express social capabilities to perform distinct personalities, develop social behaviors, and perceive children's feelings. When dealing with a learning delay, the intelligence of the robot gets relevant.

The risk of overloading information. Robots were accessible to all children, with numerous sensors and multimodal feedback, allowing children to access information simultaneously and choose the preferred way to interact. Our research recommends these multiple ways of interacting and enhancing the robot with multiple assistive tools, allowing an all in one solution to interact anywhere. However, this recommendation brings other challenges that need to be addressed. When designing with multiple communication modalities, there is a risk of overloading information to the class [22], creating a noisy interaction instead of a clear one, promoting a distraction instead of engagement in classroom activities. Another challenge is in the robot input information. With several sensors and multiple children interacting simultaneously, robots can be confused by the amount of information received. It can be challenging to identify to whom and when to interact. Therefore, we recommend a balanced use of communication channels and less intrusive modalities to interact with the Robot, as using colors, lights, gestures, sound bites, and pressure.

Robot can also be an intruder. One implication of using robots in an inclusive classroom is their distraction factor of its performance in classroom dynamics. During role-plays, children acted with robots in a controlled way. They decided when the robot should speak, move, or provide any service, in a predefined screenplay. However, in a real classroom environment, the intended robot autonomy and dynamism, by deciding how to behave, when to move, and circulate, can be a barrier for inclusion. Robots can distract the children's from class, just by moving around and acting when not expected. In future designs, we recommend designers to tune robot autonomy to avoid this distraction effect.

\subsection{Broader Implications for Inclusive Classrooms}

It was hard to define inclusion at school without creating a children's stigmatization feeling. Researchers and educators decided to define inclusive activity as a group activity accessible to all. Based on this definition, children explored several types of exclusion: shyness, loneliness, impairment, no technology access, and mastery of school subjects. Young children do not perceive impairment as exclusion at all, and only in the eighth grade class, the teams addressed it thought their robots. However, all grades children add accessibility features in their robots. When designing for inclusion between children, we suggest that the definition of inclusion should be crystal clear without being a segregation factor.

\subsection{Limitations and Future Work}

Our study has several limitations, we worked with one private inclusive schools with few visually impaired children, and this school community focused on inclusive teaching with sighted and visually impaired children.
Because the last three sessions of the participatory design were remote, we had several limitations : primary school teams were not able to conclude their work; Older children worked and talked with each other asynchronously. Without observation, findings from those conversations were not explored or recorded; and Family members may have influenced the final work. Although, we never felt participants were restricted, as they were highly engaged through all the workshops and develop new ideas.

We focused mainly on one robot's behaviors to many children; therefore, future work should explore the design patterns of inclusion in groups of mixed-visual abilities children with more than one robot. The use of social robots in classroom settings raises several ethics concerns that are being discussed in the HRI field, in our future work we intend to study the ethics and emerging tensions when deploying these technologies in the real-world contexts. We intend also to reconfirm our findings in a public schools, and build and evaluate our recommendations' effectiveness in a long-term study in classrooms and remote educational lessons.

\section{CONCLUSION}

We conducted a 4-month long community-based design research process to explore the potential of social robots in supporting inclusive classroom experiences. We engaged with community stakeholders - educators, teaching assistants, special needs professionals, parents, sighted children, and children with visual impairments - to explore the barriers to inclusion in schools and how robots could help overcome these barriers. Findings highlight issues with group participation, nonverbal communication, access to information, attitudes towards disability, and task execution. We then embedded a participatory design process within the school curricula and engaged children to reflect how social robots could be used in a set of classroom activities. Outcomes include recommendations for future design social robots area within mixed-visual abilities classrooms. Our recommendations emphasize the importance of physicality, spatial navigation, affective capabilities, and multisensory feedback as a means to support social engagement and accessibility.

\section{ACKNOWLEDGMENTS}

We would like to thank the staff and children of Centro Helen Keller for their participation, support, and understanding through these studies. National funds have supported this work through FCT, Fundação para a Ciência e a Tecnologia, under projects UIDB/50021/2020 and UIDB/50009/2020.

\section{REFERENCES}

[1] Muneeb Imtiaz Ahmad, Omar Mubin, and Joanne Orlando. 2017. Adaptive social robot for sustaining social engagement during long-term children-robot interaction. International fournal of Human-Computer Interaction 33, 12 (2017), 943-962.

[2] Dragan Ahmetovic, Cole Gleason, Chengxiong Ruan, Kris Kitani, Hironobu Takagi, and Chieko Asakawa. 2016. NavCog: A Navigational Cognitive Assistant for the Blind. In Proceedings of the 18th International Conference on HumanComputer Interaction with Mobile Devices and Services (Florence, Italy) (MobileHCI '16). Association for Computing Machinery, New York, NY, USA, 90-99. https: //doi.org/10.1145/2935334.2935361

[3] Patricia Alves-Oliveira. 2020. Boosting Children's Creativity through Creative Interactions with Social Robots. Ph.D. Dissertation. University Institute of Lisbon, School of Social Sciences and Humanities, Lisbon, Portugal. https://repositorio. iscte-iul.pt/handle/10071/20620 
[4] Patrícia Alves-Oliveira, Patrícia Arriaga, Ana Paiva, and Guy Hoffman. 2017. YOLO, a Robot for Creativity: A Co-Design Study with Children. In Proceedings of the 2017 Conference on Interaction Design and Children (Stanford, California, USA) (IDC '17). Association for Computing Machinery, New York, NY, USA, 423-429. https://doi.org/10.1145/3078072.3084304

[5] Anki. 2016. Cozmo robot. https://www.digitaldreamlabs.com/pages/cozmo

[6] Tamarah M Ashton. 2002. Assistive technology. Fournal of Special Education Technology 18, 1 (2002), 39-43.

[7] Thibault Asselborn, Arzu Guneysu, Khalil Mrini, Elmira Yadollahi, Ayberk Ozgur Wafa Johal, and Pierre Dillenbourg. 2018. Bringing Letters to Life: Handwriting with Haptic-Enabled Tangible Robots. In Proceedings of the 17th ACM Conference on Interaction Design and Children (Trondheim, Norway) (IDC '18). Association for Computing Machinery, New York, NY, USA, 219-230. https://doi.org/10. $1145 / 3202185.3202747$

[8] S. Azenkot, C. Feng, and M. Cakmak. 2016. Enabling building service robots to guide blind people a participatory design approach. In 2016 11th ACM/IEEE International Conference on Human-Robot Interaction (HRI). IEEE, Association for Computing Machinery, New York, NY, USA, 3-10. https://doi.org/10.1109/HRI 2016.7451727

[9] Kim Baraka, Patrícia Alves-Oliveira, and Tiago Ribeiro. 2020. An extended framework for characterizing social robots. In Human-Robot Interaction. Springer Springer, Cham, 21-64.

[10] Sandra Bardot, Marcos Serrano, Bernard Oriola, and Christophe Jouffrais. 2017. Identifying How Visually Impaired People Explore Raised-Line Diagrams to Improve the Design of Touch Interfaces. In Proceedings of the 2017 CHI Conference on Human Factors in Computing Systems (Denver, Colorado, USA) (CHI '17). Association for Computing Machinery, New York, NY, USA, 550-555. https: //doi.org/10.1145/3025453.3025582

[11] Renata Pitta Barros, Aquiles Medeiros Filgueira Burlamaqui, Samuel Oliveira de Azevedo, Sarah Thomaz de Lima Sa, Luiz Marcos Garcia Goncalves, Akynara Aglae R S da Silva, et al. 2017. Cardbot-assistive technology for visually impaired in educational robotics: Experiments and results. IEEE Latin America Transactions 15, 3 (2017), 517-527.

[12] Tony Belpaeme, James Kennedy, Aditi Ramachandran, Brian Scassellati, and Fumihide Tanaka. 2018. Social robots for education: A review. Science robotics 3 , 21 (2018).

[13] Mayara Bonani, Raquel Oliveira, Filipa Correia, André Rodrigues, Tiago Guerreiro, and Ana Paiva. 2018. What My Eyes Can't See, A Robot Can Show Me: Exploring the Collaboration Between Blind People and Robots. In Proceedings of the 20th International ACM SIGACCESS Conference on Computers and Accessibility (Galway, Ireland) (ASSETS '18). Association for Computing Machinery, New York, NY, USA 15-27. https://doi.org/10.1145/3234695.3239330

[14] Emily C Bouck. 2016. A national snapshot of assistive technology for students with disabilities. Fournal of Special Education Technology 31, 1 (2016), 4-13.

[15] Virginia Braun and Victoria Clarke. 2014. What can "thematic analysis" offer health and wellbeing researchers? International journal of qualitative studies on health and well-being 9 (2014).

[16] David J Brown and Michael J Proulx. 2016. Audio-vision substitution for blind individuals: Addressing human information processing capacity limitations. IEEE Journal of Selected Topics in Signal Processing 10, 5 (2016), 924-931.

[17] Emeline Brule, Gilles Bailly, Anke Brock, Frederic Valentin, Grégoire Denis, and Christophe Jouffrais. 2016. MapSense: Multi-Sensory Interactive Maps for Children Living with Visual Impairments. In Proceedings of the 2016 CHI Conference on Human Factors in Computing Systems (San Jose, California, USA) (CHI '16). Association for Computing Machinery, New York, NY, USA, 445-457. https://doi.org/10.1145/2858036.2858375

[18] Colin Burns, Eric Dishman, William Verplank, and Bud Lassiter. 1994. Actors, hairdos \& videotape-informance design. In Conference companion on Human factors in computing systems. ACM, New York, NY, USA, 119-120.

[19] Kathy Charmaz. 2006. Constructing grounded theory: A practical guide through qualitative analysis. Sage Publications, London, England.

[20] Paul T Costa Jr and Robert R McCrae. 2008. The Revised NEO Personality Inventory (NEO-PI-R). Sage Publications, Inc, Los Angeles, CA.

[21] Clare Cullen and Oussama Metatla. 2019. Co-Designing Inclusive Multisensory Story Mapping with Children with Mixed Visual Abilities. In Proceedings of the 18th ACM International Conference on Interaction Design and Children (Boise, ID, USA) (IDC '19). Association for Computing Machinery, New York, NY, USA, 361-373. https://doi.org/10.1145/3311927.3323146

[22] Luigi F Cuturi, Elena Aggius-Vella, Claudio Campus, Alberto Parmiggiani, and Monica Gori. 2016. From science to technology: Orientation and mobility in blind children and adults. Neuroscience \& Biobehavioral Reviews 71 (2016), 240-251.

[23] Kerstin Dautenhahn. 2003. Roles and functions of robots in human society: implications from research in autism therapy. Robotica 21, 4 (2003), 443.

[24] Gareth Davies. 2019. Support for pupils with special educational needs and disabilities in England: report by the Comptroller and Auditor General. National Audit Office 1, 1 (2019), 1-60.

[25] Maria Chiara Di Lieto, Emanuela Castro, Chiara Pecini, Emanuela Inguaggiato, Francesca Cecchi, Paolo Dario, Giovanni Cioni, and Giuseppina Sgandurra. 2019.
Improving Executive Functions at School in Children With Special Needs by Educational Robotics. Frontiers in Psychology 10 (2019).

[26] Julie Ducasse, Anke M Brock, and Christophe Jouffrais. 2018. Accessible interactive maps for visually impaired users. In Mobility of visually impaired people. Springer, Cham, 537-584.

[27] Efthymia Efthymiou and Alison Kington. 2017. The development of inclusive learning relationships in mainstream settings: A multimodal perspective. Cogent Education 4, 1 (2017), 1304015.

[28] Paul Ekman. 1999. Basic emotions. Handbook of cognition and emotion 98, 45-60 (1999), 16.

[29] Paul Ekman and Daniel Cordaro. 2011. What is meant by calling emotions basic. Emotion review 3, 4 (2011), 364-370.

[30] Terrence Fong, Illah Nourbakhsh, and Kerstin Dautenhahn. 2003. A survey of socially interactive robots. Robotics and autonomous systems 42, 3-4 (2003), 143-166.

[31] Dario Galati, Renato Miceli, and Barbara Sini. 2001. Judging and coding facial expression of emotions in congenitally blind children. International fournal of Behavioral Development 25, 3 (2001), 268-278. https://doi.org/10.1080/ 01650250042000393

[32] Patrick GUNKEL. 1998. Positive and negative traits. http://ideonomy.mit.edu/ essays/traits.html

[33] Patrick GUNKEL. 2013. 638 Primary Personality Traits. Ideonomy: The Science of Ideas 1,1 (2013)

[34] Deanna Hood, Séverin Lemaignan, and Pierre Dillenbourg. 2015. When children teach a robot to write: An autonomous teachable humanoid which uses simulated handwriting. In Proceedings of the Tenth Annual ACM/IEEE International Conference on Human-Robot Interaction. Association for Computing Machinery, New York, NY, USA, 83-90.

[35] Seray B Ibrahim, Asimina Vasalou, and Michael Clarke. 2020. Can design documentaries disrupt design for disability?. In Proceedings of the Interaction Design and Children Conference. Association for Computing Machinery, New York, NY, USA, 96-107.

[36] iRobot. 2000. Roomba robot. https://www.irobot.com/roomba/i-series

[37] Frank Jamet, Olivier Masson, Baptiste Jacquet, Jean-Louis Stilgenbauer, and Jean Baratgin. 2018. Learning by teaching with humanoid robot: a new powerful experimental tool to improve children's learning ability. fournal of Robotics 2018 (2018).

[38] Wafa Johal. 2020. Research Trends in Social Robots for Learning. Current Robotics Reports June 2020 (2020), 1-9.

[39] Hatice Köse, Pınar Uluer, Neziha Akalın, Rabia Yorgancı, Ahmet Özkul, and Gökhan Ince. 2015. The effect of embodiment in sign language tutoring with assistive humanoid robots. International Journal of Social Robotics 7, 4 (2015), 537-548.

[40] Aditi Kulkarni, Allan Wang, Lynn Urbina, Aaron Steinfeld, and Bernardine Dias. 2016. Robotic assistance in indoor navigation for people who are blind. In 2016 11th ACM/IEEE International Conference on Human-Robot Interaction (HRI). IEEE, Association for Computing Machinery, New York, NY, USA, 461-462.

[41] Innvo Labs. 2006. Pleo robot. https://www.pleoworld.com/pleo_rb/eng/products. php?c1id=1

[42] Hee Rin Lee, Selma Šabanović, Wan-Ling Chang, Shinichi Nagata, Jennifer Piatt, Casey Bennett, and David Hakken. 2017. Steps toward participatory design of social robots: mutual learning with older adults with depression. In Proceedings of the 2017 ACM/IEEE international conference on human-robot interaction. Association for Computing Machinery, New York, NY, USA, 244-253.

[43] Lego. 2013. Mindstorms robot. https://www.lego.com/en-gb/themes/mindstorms

[44] Alexandru Litoiu and Brian Scassellati. 2015. Robotic coaching of complex physical skills. In Proceedings of the Tenth Annual ACM/IEEE International Conference on Human-Robot Interaction Extended Abstracts. Association for Computing Machinery, New York, NY, USA, 211-212.

[45] Anne-Marie Mann, Uta Hinrichs, Janet C Read, and Aaron Quigley. 2016. Facilitator, functionary, friend or foe? Studying the role of iPads within learning activities across a school year. In Proceedings of the 2016 CHI Conference on Human Factors in Computing Systems. Association for Computing Machinery, New York, NY, USA, 1833-1845

[46] Laura Martinez, Virginia B Falvello, Hillel Aviezer, and Alexander Todorov. 2016. Contributions of facial expressions and body language to the rapid perception of dynamic emotions. Cognition and Emotion 30, 5 (2016), 939-952.

[47] Samantha McDonald, Joshua Dutterer, Ali Abdolrahmani, Shaun K Kane, and Amy Hurst. 2014. Tactile aids for visually impaired graphical design education. In Proceedings of the 16th international ACM SIGACCESS conference on Computers \& accessibility. Association for Computing Machinery, New York, NY, USA, 275276.

[48] Oussama Metatla. 2017. Uncovering challenges and opportunities of including children with visual impairments in mainstream schools. Electronic Visualisation and the Arts (EVA 2017) 1 (2017), 1-6.

[49] Oussama Metatla, Sandra Bardot, Clare Cullen, Marcos Serrano, and Christophe Jouffrais. 2020. Robots for Inclusive Play: Co-designing an Educational Game With Visually Impaired and sighted Children. In Proceedings of the $2020 \mathrm{CHI}$ 
Conference on Human Factors in Computing Systems. Association for Computing Machinery, New York, NY, USA, 1-13.

[50] Oussama Metatla, Nick Bryan-Kinns, Tony Stockman, and Fiore Martin. 2015 Designing with and for people living with visual impairments: audio-tactile mock-ups, audio diaries and participatory prototyping. CoDesign 11, 1 (2015), $35-48$.

[51] Oussama Metatla and Clare Cullen. 2018. "Bursting the Assistance Bubble" Designing Inclusive Technology with Children with Mixed Visual Abilities. In Proceedings of the 2018 CHI Conference on Human Factors in Computing Systems. Association for Computing Machinery, New York, NY, USA, 1-14.

[52] Oussama Metatla, Alison Oldfield, Taimur Ahmed, Antonis Vafeas, and Sunny Miglani. 2019. Voice user interfaces in schools: Co-designing for inclusion with visually-impaired and sighted pupils. In Proceedings of the 2019 CHI Conference on Human Factors in Computing Systems. Association for Computing Machinery, New York, NY, USA, 1-15.

[53] Oussama Metatla, Janet C Read, and Matthew Horton. 2020. Enabling children to design for others with expanded proxy design. In Proceedings of the Interaction Design and Children Conference. Association for Computing Machinery, New York, NY, USA, 184-197.

[54] Oussama Metatla, Marcos Serrano, Christophe Jouffrais, Anja Thieme, Shaun Kane, Stacy Branham, Émeline Brulé, and Cynthia L Bennett. 2018. Inclusive education technologies: Emerging opportunities for people with visual impairments. In Extended Abstracts of the 2018 CHI Conference on Human Factors in Computing Systems. Association for Computing Machinery, New York, NY, USA, 1-8.

[55] Oussama Metatla, Anja Thieme, Emeline Brulé, Cynthia Bennett, Marcos Serrano, and Christophe Jouffrais. 2018. Toward classroom experiences inclusive of students with disabilities. interactions 26, 1 (2018), 40-45.

[56] Lauren R Milne, Cynthia L Bennett, Richard E Ladner, and Shiri Azenkot. 2014. BraillePlay: educational smartphone games for blind children. In Proceedings of the 16th international ACM SIGACCESS conference on Computers \& accessibility. Association for Computing Machinery, New York, NY, USA, 137-144.

[57] Miguel Neiva. 2010. Color Identification System for colorblind people. Retrieved June 7, 2006 from http://www.coloradd.net/

[58] Isabel Neto, Wafa Johal, Marta Couto, Hugo Nicolau, Ana Paiva, and Arzu Guneysu. 2020. Using tabletop robots to promote inclusive classroom experiences In Proceedings of the Interaction Design and Children Conference. Association for Computing Machinery, New York, NY, USA, 281-292.

[59] A. T. Noman, M. A. M. Chowdhury, H. Rashid, S. M. S. R. Faisal, I. U. Ahmed, and S. M. T. Reza. 2017. Design and implementation of microcontroller based assistive robot for person with blind autism and visual impairment. In 2017 20th International Conference of Computer and Information Technology (ICCIT). IEEE, Los Alamitos, CA, 1-5. https://doi.org/10.1109/ICCITECHN.2017.8281806

[60] Mohammad Obaid, Asım Evren Yantaç, Wolmet Barendregt, Güncel Kırlangıç, and Tilbe Göksun. 2016. Robo2Box: a toolkit to elicit children's design requirements for classroom robots. In International Conference on Social Robotics. Springer, Springer, Cham, 600-610.

[61] Ayberk Òzgür, Séverin Lemaignan, Wafa Johal, Maria Beltran, Manon Briod, Léa Pereyre, Francesco Mondada, and Pierre Dillenbourg. 2017. Cellulo: Versatile handheld robots for education. In 2017 12th ACM/IEEE International Conference on Human-Robot Interaction (HRI. IEEE, Association for Computing Machinery, New York, NY, USA, 119-127.
[62] Beryl Plimmer, Andrew Crossan, Stephen A Brewster, and Rachel Blagojevic. 2008. Multimodal collaborative handwriting training for visually-impaired people. In Proceedings of the SIGCHI Conference on Human Factors in Computing Systems. Association for Computing Machinery, New York, NY, USA, 393-402.

[63] S Prasanna, N Priyadharshini, and M Arul Pugazhendhi. 2017. Textile robot for matching and pick up clothes based on color recognition. Asian fournal of Applied Science and Technology (AJAST) 1, 3 (2017), 62-65.

[64] Peter Reid and Beryl Plimmer. 2008. A Collaborative Multimodal Handwriting Training Environment for Visually Impaired Students. In Proceedings of the 20th Australasian Conference on Computer-Human Interaction: Designing for Habitus and Habitat (Cairns, Australia) (OZCHI '08). Association for Computing Machinery, New York, NY, USA, 195-202. https://doi.org/10.1145/1517744.1517808

[65] Philips research. 2007. iCat robot. https://www.technologyreview.com/2007/07/ 26/224540/an-emotional-cat-robot/

[66] Wyca robotics. 2015. Keylo robot. https://www.wyca-robotics.com/

[67] Kasper Rodil, Matthias Rehm, and Antonia Lina Krummheuer. 2018. Co-Designing Social Robots with Cognitively Impaired Citizens. In Proceedings of the 10th Nordic Conference on Human-Computer Interaction (Oslo, Norway) (NordiCHI '18). Association for Computing Machinery, New York, NY, USA, 686-690. https: //doi.org/10.1145/3240167.3240253

[68] Joao Roe. 2008. Social inclusion: meeting the socio-emotional needs of children with vision needs. British fournal of Visual Impairment 26, 2 (2008), 147-158.

[69] Nuzhah Gooda Sahib, Tony Stockman, Anastasios Tombros, and Oussama Metatla. 2013. Participatory design with blind users: a scenario-based approach. In IFIP Conference on Human-Computer Interaction. Springer, Springer, Cham, 685-701.

[70] Brian Scassellati, Laura Boccanfuso, Chien-Ming Huang, Marilena Mademtzi, Meiying Qin, Nicole Salomons, Pamela Ventola, and Frederick Shic. 2018. Improving social skills in children with ASD using a long-term, in-home social robot. Science Robotics 3, 21 (2018)

[71] Dennis Schleicher, Peter Jones, and Oksana Kachur. 2010. Bodystorming as embodied designing. Interactions 17, 6 (2010), 47-51.

[72] Softbankrobotics. 2006. Nao robot. https://www.softbankrobotics.com/emea/ en/nao

[73] Sphero. 2013. Sphero robot. https://sphero.com/

[74] Xiang Zhi Tan and Aaron Steinfeld. 2017. Using Robot Manipulation to Assist Navigation by People Who Are Blind or Low Vision. In Proceedings of the Companion of the 2017 ACM/IEEE International Conference on Human-Robot Interaction (Vienna, Austria) (HRI '17). Association for Computing Machinery, New York, NY, USA, 379-380. https://doi.org/10.1145/3029798.3034808

[75] Anja Thieme, Cecily Morrison, Nicolas Villar, Martin Grayson, and Siân Lindley. 2017. Enabling Collaboration in Learning Computer Programing Inclusive of Children with Vision Impairments. In Proceedings of the 2017 Conference on Designing Interactive Systems (Edinburgh, United Kingdom) (DIS '17). Association for Computing Machinery, New York, NY, USA, 739-752. https://doi.org/10. $1145 / 3064663.3064689$

[76] Make Wonder. 2013. Dash robot. https://www.makewonder.com/

[77] Sarah Woods. 2006. Exploring the design space of robots: Children's perspectives. Interacting with Computers 18, 6 (2006), 1390-1418.

[78] Anon Ymous, Katta Spiel, Os Keyes, Rua M Williams, Judith Good, Eva Hornecker, and Cynthia L Bennett. 2020. " I am just terrified of my future"-Epistemic Violence in Disability Related Technology Research. In Extended Abstracts of the 2020 CHI Conference on Human Factors in Computing Systems. Association for Computing Machinery, New York, NY, USA, 1-16. 